\title{
From Newcastle MOUTH to Aussie ears: Australians' perceptual assimilation and adaptation for Newcastle UK vowels
}

\author{
${ }^{1}$ University of Western Sydney, Australia \\ ${ }^{2}$ Griffith University, Australia \\ ${ }^{3}$ University College London, UK \\ ${ }^{4}$ University of York, UK \\ ${ }^{5}$ University of Canterbury, NZ \\ ${ }^{6}$ Newcastle University, UK \\ C. best@uws . edu . au
}

Catherine T. Best ${ }^{1}$, Jason A. Shaw ${ }^{1}$, Gerard Docherty ${ }^{2}$, Bronwen G. Evans ${ }^{3}$, Paul Foulkes 4 , Jennifer Hay ${ }^{5}$, Jalal Al-Tamimi ${ }^{6}$, Katharine Mair ${ }^{3}$, Karen E. Mulak ${ }^{1}$, Sophie Wood ${ }^{4}$

\begin{abstract}
To probe how episodic and abstract processes contribute to flexible perception of phonetically variable speech, we evaluated Australian (Aus) listeners' perception of Aus-accented vowels versus those of an unfamiliar accent: Newcastle UK $(\mathrm{Ncl})$. Aus listeners first heard a round-robin story told by multiple talkers of Aus or $\mathrm{Ncl}$, then categorized multi-talker tokens of 20 vowels in nonce words spoken in the Aus or Ncl accent. Categorization was variable even across Aus nonce vowels ( $M_{\text {accuracy }}$ ranged from $21-80 \%$ ). Perceptual assimilation of $\mathrm{Ncl}$ vowels (Aus passage $/ \mathrm{Ncl}$ nonce) was diverse: Some were categorized very much like the corresponding Aus vowel. Some showed within-category differentiation from Aus; others were heard as a different vowel altogether. Perception of some $\mathrm{Ncl}$ vowels changed after $\mathrm{Ncl}$ passage exposure, including both positive adaptation (improved categorization: e.g., MOUTH, FLEECE, TRAP) and negative shifts (increased differentiation from the corresponding Aus vowel: e.g., NURSE, FOOT). Assimilation and adaptation patterns were largely consistent with similarities and dissimilarities between the Aus and $\mathrm{Ncl}$ vowel spaces. Implications of the results for episodic and abstract contributions to perceptual flexibility are discussed. We also consider the possibility that listeners perceptually adjust to other-accent vowels as a system, rather than treating each vowel as an independent entity.
\end{abstract}

Index Terms: regional accents, vowel perception, crossaccent perceptual assimilation, multi-talker accent adaptation

\section{Introduction}

A core debate over perception of spoken words is how abstract knowledge about their phonological composition [1] and episodic memory for specific tokens of a word interact to support flexible word recognition [2,3]. Listeners familiarized to words spoken with "odd" variants of one vowel/contrast generalize the deviant variants to recognition of untrained words by the same speaker that contain those vowels [4-6]. This indicates that phonemes can be a locus of perceptual adaptation, but does not reveal how episodic learning contributes. Moreover, such localized variations may not reveal whether, or how, episodic and abstract processes support adaptation to more systemic variations including those found in the vowel systems of other accents of the language. Indeed, accent variation initially impairs comprehension of speech in noise $[7,8]$, but listeners rapidly adapt to unfamiliar accents, showing reduced difficulty in understanding accented speech in noise [9-12]. But these studies tested comprehension of running speech. To address how episodic and abstract processes combine to effect perceptual adaptation to an unfamiliar accent, we need evidence on how phonological segments are perceived before and after exposure to it.

We exploited natural variation between the vowel systems of two regional English accents to address this issue. Specifically, we assessed how Australian English (Aus) listeners perceive vowels from an accent that is unfamiliar to them: the variety spoken around Newcastle UK $(\mathrm{Ncl})$ [see 13].

We use Wells' [14] very useful terminology for English accent descriptions. Aus and Ncl show two systemic differences. First, Ncl lacks the FOOT-STRUT lexical set split found in Aus. In northern England, including Ncl, the STRUT vowel is realized with the same quality as FOOT [U], i.e., lower F1 and F2 than Aus STRUT; it overlaps FOOT in both accents. Second, $\mathrm{Ncl}$ realizes the restricted lexical set BATH with the same vowel as TRAP, [æ/a] (high F1, mid-range F2). Aus BATH is instead realised with the vowel quality of Aus PALM ([e:] (lower F2 than TRAP). Also, in recent generations Aus TRAP has moved toward [a] (higher F1) [15]. Ncl TRAP/BATH falls in-between Aus TRAP and BATH/PALM (see Figure 1).

Other vowels show Ncl realizational differences from Aus that are sufficiently disparate as to result in misperception, or Category Shifts (CS), by Aus listeners. For example, Ncl GOAT is variably realised as [0:]/[Uə]. The [o:] variant is likely to be misheard by Aus listeners as THOUGHT, the [Uə] as a disyllabic vowel found in some CURE words (e.g., <tour>, see below). Ncl PRICE is realised as a rising diphthong, occasionally with a higher, fronted onset ([EI], lower F1) than Aus, which might be miscategorised as FACE.

But other realization differences may be heard as a goodness of fit variation from the Aus vowel (Category Goodness difference: $C G$ ), e.g., $\mathrm{Ncl}$ MOUTH, a rising diphthong with a larger F1 trajectory ([av]/[ev]) than Aus MOUTH ([æə]), is likely to sound qualitatively different from Aus, but unlikely be categorically misperceived. The remaining Ncl lexical set vowels are likely either to be perceived as a $C G$ difference from Aus, or to be heard by Aus listeners as Native-Like (NL), as their realizations are similar to Aus, e.g., FOOT, CHOICE.

The status of CURE in both Aus and $\mathrm{Ncl}$ deserves comment. The diphthongal variant is produced infrequently by 
younger Aus speakers, who more often produce a monophthongal [o:], merged with THOUGHT, or sometimes a disyllabic [ $\mathrm{H}: \partial]$ [16]. Ncl has a similar range of variation, but its younger speakers quite commonly produce diphthongal and disyllabic variants. Thus, CURE may be another systemic difference for younger Aus vs Ncl speakers, but it is not as entrenched as the FOOT-STRUT or TRAP/BATH/PALM cases.

To assess perceptual effects of Aus-Ncl vowel differences, we adapted the perceptual assimilation task from crosslanguage speech perception research, asking listeners to categorize the vowels of nonce words in one of the accents [17, 18]. This task requires phonological judgments, isolating the perceptual effects at the vowel phoneme level. In order to assess whether prior exposure to Aus or the other accent results in perceptual adaptation to that accent, and thereby influences the vowel categorizations in the assimilation task, listeners first heard a story told by speakers of a given accent. Examining Aus listeners' perceptual responses to the full $\mathrm{Ncl}$ vowel system, rather than to only a small number of $\mathrm{Ncl}$ vowels or to the global intelligibility of connected speech (in noise), is likely to offer novel insights into how listeners adapt to another regional accent of their language. Such data may reveal whether they adapt to each phoneme independently, or instead make more systemic adjustments in the vowel space.

\section{Method}

\subsection{Participants}

48 undergraduates (University of Western Sydney) aged 17.930.2 years $(M=21.1 ; S D=2.7)$ participated as listeners for course credit. All were native monolingual Aus speakers without hearing/language problems and no regular exposure to other languages or other regional English accents. All were raised in monolingual Aus homes in Greater Western Sydney.

\subsection{Stimuli}

\subsubsection{Nonce words}

The frame $/ \mathbf{z} \mathbf{V} b a /$ was used to generate nonce words for each of the English vowels in 20 lexical sets [14]: FLEECE, KIT, NEAR, DRESS, SQUARE, TRAP, PALM, STRUT, GOOSE, FOOT, CURE, THOUGHT, LOT, NURSE, FACE, GOAT, PRICE, CHOICE, MOUTH, and BATH (a restricted lexical set, same vowel as PALM in Aus but the same as TRAP vowel in Ncl). None of the resulting nonce items is a real word. The low phonotactic probability of $/ \mathrm{zVb} /$ also minimizes lexical biases in perception of the nonce target items.

The nonce targets were produced six times by two female and two male speakers from each of Greater Western Sydney (Aus: 17.0-26.4 years, $M=21.7, S D=3.9$ ), and the Newcastle area (Ncl: $21.5-45.9$ years, $M=31.6, S D=11.7)$. Two tokens per nonce word per speaker were selected on the basis that the target vowel was judged satisfactory for the accent by a phonetically-trained researcher experienced with that accent. Tokens were extracted with a $100 \mathrm{~ms}$ buffer at the beginning and end. A ramp and damp were imposed on the initial and final $20 \mathrm{~ms}$ of each file, and tokens were normalized to $65 \mathrm{~dB}$.

\subsubsection{Accent exposure passage}

We generated a version of the children's story Chicken Little that contained at least ten occurrences, in stressed syllables of real words, of each vowel used in the nonce items. The exposure passage for our study used recordings of this story by two male and two female Aus speakers from greater Western
Sydney (18.8-43.9 yr, $M=31.8, S D=10.3)$ and two male and two female $\mathrm{Ncl}$ speakers (20.4-37.8 yr, $M=26.2, S D=7.9$ ). None were the same speakers as used for the nonce tokens.

The exposure passage was created separately for Aus and $\mathrm{Ncl}$ by selecting three non-adjacent subsections of the passage for each of the four speakers of that accent, and concatenating the subsections in sequence to form a complete story. A $1.5 \mathrm{~s}$ fade out and fade in was added between subsections (corresponding to the natural pauses speakers left between subsections). The final passage was scaled to $65 \mathrm{~dB}$.

\subsubsection{Keywords}

For 19 of the target vowels, a real word served as a printed keyword for the assimilation task (we excluded a BATH keyword, as its vowel groups with Aus PALM but Ncl TRAP). Keywords were generally of the form $/ b \underline{V} d /$, though exceptions were made when $/ \mathrm{b} \underline{\mathbf{V}} \mathrm{d} / \mathrm{did}$ not yield an easily recognized word (e.g., $<$ code $>$ instead of $<$ bode $>$; keywords $=$

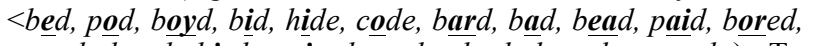
proud, hood, bird, paired, rude, bud, beard, toured $>$ ). To confirm that our speakers' vowels were representative of the accent descriptions, we analyzed keyword recordings by the Aus and Ncl nonce talkers (3-5 tokens/word/talker). We used Lobanov-normalized F1/F2 values [19] (z-scores of each speaker's formant values projected on a common F1/F2 space) to estimate their vowel spaces (Figure 1). Note that Aus and $\mathrm{Ncl}$ differ as to which vowels are realized as diphthongs (red arrows; F1 and/or F2 change over $20-80 \%$ of the vowel). Both accents have some dense regions of overlapping vowels, and sparser regions with little or no overlap, but they differ somewhat in those vowel groupings. The alignments/misalignments between $\mathrm{Ncl}$ and Aus vowels are largely consistent with our earlier descriptions. Note, though, that categorization and ratings of vowels in both accents will also be affected by (near-)mergers (e.g., Aus NEAR-FLEECE), and by properties not captured by $\mathrm{F} 1 / \mathrm{F} 2$ plots (tense lax/duration distinction between vowels of highly overlapping F1/F2 locations).

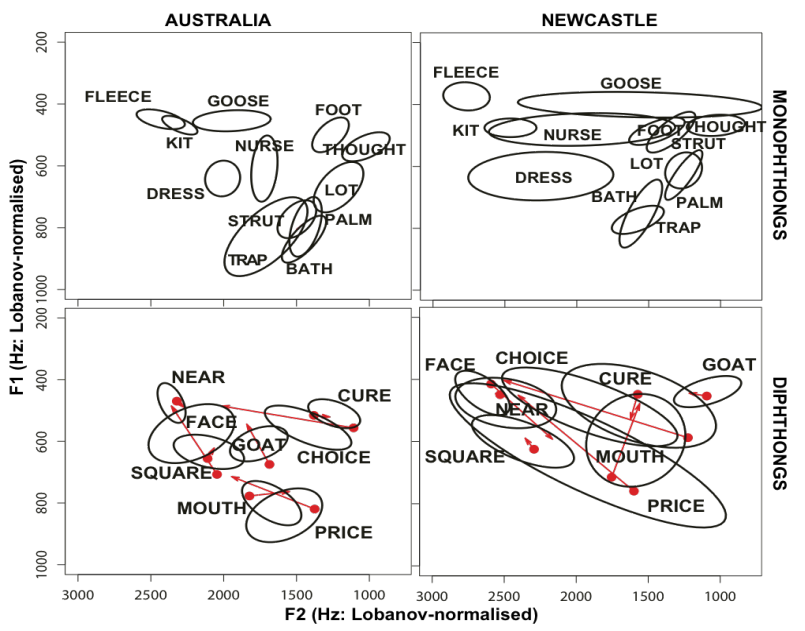

Figure 1. Aus and Ncl nonce keyword lexical set vowels for monophthongs vs diphthongs. Ellipses $=F 1 / F 2$ at $50 \%$; red arrows $=20 \rightarrow 80 \%$ change in diphthongs.

\subsection{Procedure}

Participants were assigned to one of three conditions: Aus exposure story/Aus nonce words (A-A), Aus story/ $\mathrm{Ncl}$ nonce $(\mathrm{A}-\mathrm{N})$, or $\mathrm{Ncl}$ story/Ncl nonce $(\mathrm{N}-\mathrm{N})$. They first completed an exposure phase; they listened to the Chicken Little story in the accent for their condition, either A or N, then answered five 
multiple choice questions about the story to ensure they had paid attention. Next, they completed the phoneme categorization task in the nonce accent for their condition (A or N).

On each trial, participants heard a nonce token. They then saw a grid on a computer monitor containing the vowel keywords. Participants clicked on the keyword whose highlighted vowel best matched the target vowel in the nonce token they had heard. The layout of keywords on the grid was randomized across participants, but the order for a given participant remained constant throughout the task. To familiarize participants with the task and their randomized grid, prior to the categorization task, they completed training trials with nonce tokens produced by the speakers of the Aus story, arranged so that they received one token per grid item. After training, participants completed the categorization test (160 trials $=20$ nonce words $\times 2$ tokens $\times 4$ speakers), presented in random order via E-Prime (v. 2.0.8.22).

\section{Results}

To evaluate accent effects, A-A is compared with A-N; to evaluate exposure effects, A-N is compared with N-N. Figure 2 shows the lexical set vowels listeners selected for each Aus nonce word; the correct choice is bold outlined. Accuracy on Aus nonce vowels is the comparison baseline. It was quite variable and never approached ceiling $(M=21-80 \%)$, consistent with the non-categorical nature of vowel perception [20], though it was well above chance $(1 / 19=5.26 \%)$ for each nonce vowel. Only 11 Aus vowels were Categorized correctly by a lenient categorization criterion of $>50 \%$ choices of a single keyword for a given vowel [21]: DRESS (80\%), LOT $(80 \%)$, CHOICE (73\%), KIT (70\%), GOAT (66\%), PRICE (66\%), PALM (63\%), BATH (identified as PALM: 63\%), TRAP (59\%), FLEECE (56\%), FACE (56\%). Four other vowels were Weakly Categorized (correct keyword chosen 35-49\%): THOUGHT $(44 \%)$, FOOT $(42 \%)$, MOUTH $(42 \%)$, NURSE $(37 \%)$. The remaining five Aus vowels were UnCategorized (two keywords were chosen roughly equivalently, each 20-35\%): SQUARE (DRESS $35 \%$, SQUARE $28 \%$ ), NEAR (NEAR 31\%, FLEECE 29\%), GOOSE (FOOT 34\%, GOOSE 31\%), STRUT (STRUT 34\%, TRAP 34\%), CURE (THOUGHT 27\%, CURE $21 \%$ ). As Figure 2 shows, for 16 nonce vowels, i.e., all except TRAP, DRESS, CHOICE and LOT, one or more, incorrect keywords were also chosen $\geq 10 \%$.

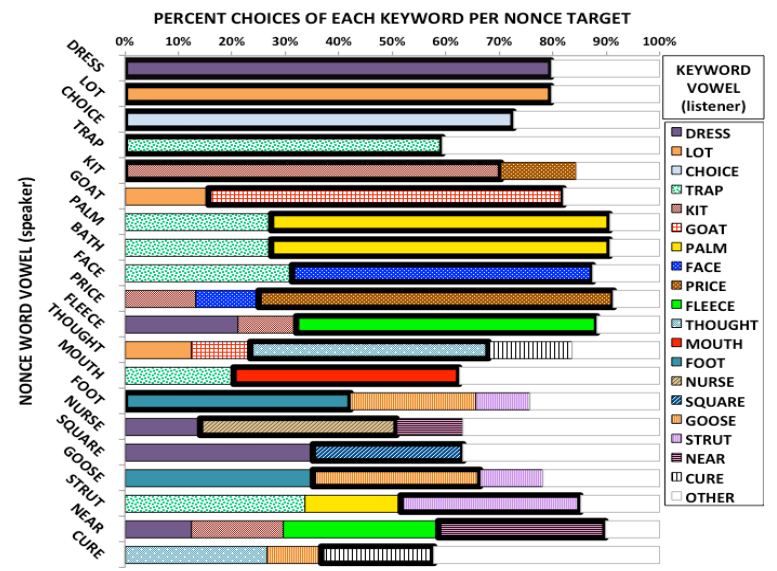

Figure 2. Categorization of native Aus vowels (A-A condition). Bold outline $=$ correct choice. OTHER $=$ sum across all keywords chosen $<5 \%$ for each target.

Thus BATH/PALM/TRAP, an Aus-Ncl systemic difference set, are all Categorized in Aus. Of these, TRAP also shows an
Aus-Ncl realization difference (lower F2: see Fig. 1). However, in the other systemic difference, STRUT/FOOT, Aus STRUT is UnCategorized, identified equally as STRUT and TRAP. Aus FOOT, though, for which the Ncl vowel is NativeLike (NL), is Weakly Categorized. But CHOICE, another Ncl vowel that is NL, is Categorized in Aus. In the possible systemic difference of CURE/THOUGHT, Aus CURE is UnCategorized; THOUGHT is Weakly Categorized. GOAT and PRICE, the two potential Category Shifting (CS) realization differences, are both Categorized in Aus. But MOUTH, an expected Category Goodness (CG) difference, is Weakly Categorized.

\subsection{Effect of Ncl accent on vowel categorization}

Figure 3 shows correct keyword categorization (accuracy) for both accents. Several vowels show lower accuracy for $\mathrm{Ncl}$ (A-N condition) than for Aus (A-A). Generalized linear mixed modeling (GLMM) in R (version 3.0.2) was used to identify statistically significant effects. We fit a mixed model to the accuracy data in A-A and A-N, using the glmer function (binomial family). Fixed factors were accent (Aus or $\mathrm{Ncl}$ ) of the nonce words, the nonce vowels, and the interaction between vowel and accent. The interaction term is crucial as we predicted non-uniform effects of accent across vowels. We included random slopes (for accent) and random intercepts (for participants and tokens). Aus was the reference intercept for accent. FOOT was the reference for vowel as it was nearest to mean accuracy for all vowels in A-A and A-N, and shows negligible accuracy differences across the two accents. In the full model, Accent was not a significant predictor $(Z=.38 ; p$ $=.71$ ), but the interaction between accent and vowel was significant for 10 vowels, the lexical sets TRAP $(z=-2.92, p$ $=.01), \operatorname{BATH}(z=-3.80, p=.001), \operatorname{PALM}(z=-5.07, p=.001)$, FACE $(z=-5.04, p=.001)$, DRESS $(z=-2.33, p=.02)$, KIT $(z$ $=-5.65, p=.01)$, LOT $(z=-3.54, p=.001)$, GOAT $(z=-7.15, p$ $=.001)$, STRUT $(z=-2.15, p=.03)$, THOUGHT $(z=2.43, p$ $=.02$ ). Nine of these were negative, i.e, lower accuracy on $\mathrm{Ncl}$ than Aus vowels. However, Ncl THOUGHT was Categorised $(70 \%)$ more accurately than Aus THOUGHT, which was Weakly Categorized (44\%; Fig. 2) and overlaps with CURE (Fig. 2).

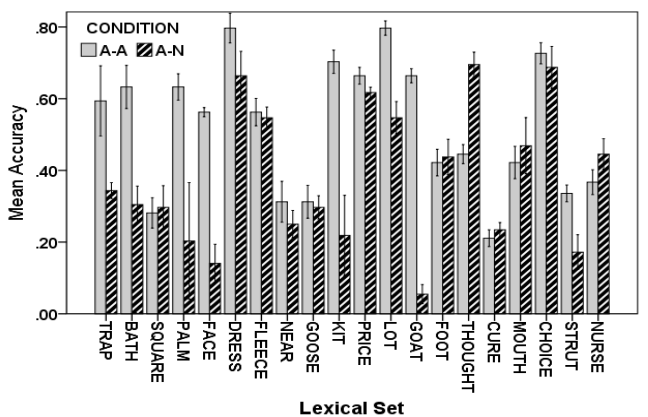

Figure 3. Categorization accuracy following the Aus story for Aus $(A-A)$ and $\mathrm{Ncl}(A-N)$ vowels. Error bars $=$ standard error of the mean (s.e.m).

To assess how listeners perceptually assimilate the vowels of other accents, however, both correct and incorrect vowel choices must be considered. In the A-N condition, which tested perceptual assimilation of $\mathrm{Ncl}$ to Aus vowels, most incorrect choices for each $\mathrm{Ncl}$ vowel were close competitors in Aus vowel space (Fig. 1). Several Ncl vowels were correctly Categorized and rated (goodness of fit) on a par with Categorized Aus vowels, i.e., were heard as Native-Like (NL): CHOICE, FLEECE, PRICE. Ncl THOUGHT and NURSE were similarly Weakly Categorised in $\mathrm{Ncl}$ and Aus. Ncl DRESS and LOT were Categorized, as in Aus, but chosen less often and/or rated as less good than in Aus, i.e., Category Goodness (CG) 
differences were perceived. MOUTH and FOOT were Weakly Categorized in both accents, but with CG differences for Ncl. Two vowels that were correctly Categorized in Aus were Categorized to incorrect vowels in $\mathrm{Ncl}$, i.e., showed a Category Shift (CS): GOAT (heard as THOUGHT), KIT (as DRESS). Categorized in Aus but Weakly Categorized and CS in Ncl were the systemic set BATH (heard as TRAP, as expected)/TRAP (as PALM)/PALM (as THOUGHT). FACE was Categorized in Aus but UnCategorized and CS in Ncl (DRESS 29\%, FLEECE 20\%). SQUARE, NEAR, CURE, STRUT, GOOSE were UnCategorized in both accents, where $\mathrm{Ncl}$ showed CS biases for STRUT (FOOT 20\%, STRUT 17\%) and NEAR (FLEECE 30\%, NEAR $25 \%$ ). Responses were more accurate for CURE in Ncl (CURE $23 \%$, THOUGHT $20 \%$ ) than Aus (THOUGHT $27 \%$, CURE $21 \%$ ).

\subsection{Effect of exposure to the Newcastle accent}

To assess how accent exposure affects vowel categorization, we compared the A-N condition (Aus story, Ncl nonce words) to the N-N condition ( $\mathrm{Ncl}$ story and nonce words). Figure 4 shows the vowel accuracy difference between the two stories. Positive bars indicate higher mean accuracy in N-N relative to the A-N baseline; negative bars indicate negative effects of $\mathrm{Ncl}$ exposure, i.e., lower accuracy in N-N than in A-N. While many vowels hover near zero (no difference across conditions), a few show large improvements after $\mathrm{Ncl}$ exposure. Listeners categorized Ncl MOUTH nearly $20 \%$ more accurately after the $\mathrm{Ncl}$ than the Aus story. There were also negative effects of $\mathrm{Ncl}$ exposure, the largest for NURSE: accuracy dropped $17.2 \%$.

We again fit a binomial mixed model to the accuracy data, this time for the A-N and N-N conditions. Fixed factors were the exposure accent (Aus, $\mathrm{Ncl}$ ), the nonce vowel, and the vowel by exposure accent interaction. Again, we applied a maximal random effects structure. The reference category (intercept) for exposure accent was Aus. The reference vowel was LOT (the most accurate Aus vowel [80\%]; Categorized in $\mathrm{Ncl}$ but at a lower level [55\%]; no exposure effects). Exposure accent was not significant as a predictor. Our primary interest, however, is the story accent by vowel interaction. There were two significant accent $*$ vowel interactions: accuracy on MOUTH $(z=2.32, p=.02)$ improved, while accuracy on NURSE declined $(z=-2.25, p=.02)$, after Ncl exposure. Accuracy improved marginally after the $\mathrm{Ncl}$ story for FLEECE $(z=1.74, p=0.08)$ and CHOICE $(z=1.74, p=0.08)$.

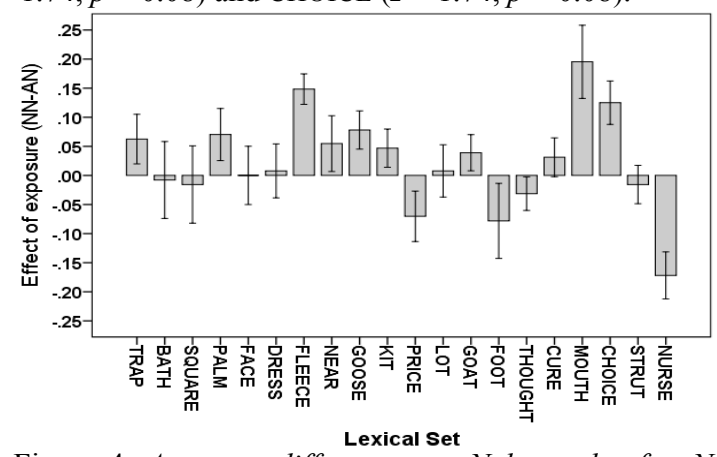

Figure 4. Accuracy differences on $\mathrm{Ncl}$ vowels after $\mathrm{Ncl}$ vs. Aus story ( $N-N$ minus $A-N)$. Error bars $=$ s.e.m.

To determine how prior exposure to $\mathrm{Ncl}$ affects perception of $\mathrm{Ncl}$ vowels, both correct and incorrect vowel choices must again be considered. The Ncl PRICE vowel shifted from being Categorized and Native-Like (NL) after the Aus story, to a more CG pattern after the $\mathrm{Ncl}$ story $(62 \rightarrow 55 \%$ correct). In addition, Ncl FOOT and NURSE shifted from being heard as NL after the Aus story, to being UnCategorized after the $\mathrm{Ncl}$ story. These changes suggest increased perceptual differentia- tion of these four $\mathrm{Ncl}$ vowels from their Aus counterparts, i.e., heightened sensitivity to fine-grained Ncl-Aus discrepancies.

Ncl MOUTH, conversely, shifted from Weakly Categorized $(47 \%)$ to Categorized (66\%). TRAP moved from UnCategorized with a CS bias (PALM 41\%, TRAP 34\%) after the Aus story, to an NL bias after the $\mathrm{Ncl}$ story (TRAP $41 \%$, PALM $35 \%)$. Both were thus heard as more similar to the Aus vowels.

\section{Discussion}

Perceptually adapting to the vowels of an unfamiliar regional accent as different as Newcastle English is to Australian English requires working out both the system of contrasts and their locations in phonetic space. From exemplar-based phonetic categorization models [e.g., 22], we can extrapolate the prediction that exposure to an accent will lead to improved categorization of its vowels. But this is not obviously supported by our findings: for some $\mathrm{Ncl}$ vowels categorization accuracy gets systematically worse, or assimilation shifts further away from the corresponding Aus vowel, after Ncl story exposure. There are more such cases than those of $\mathrm{Ncl}$ vowels that show improved accuracy or more Aus-like assimilation after $\mathrm{Ncl}$ exposure. These unexpected negative effects of $\mathrm{Ncl}$ exposure may reflect over-specific adaptation, i.e., listeners adapt to a narrow variant of a vowel rather than to its whole distribution, which could be consistent with an exemplar-based approach. For example, there is notable variation in Ncl NURSE, so our Aus listeners may have focused on one of these variants in the Ncl story, showing overly-narrow adaptation that resulted in a negative effect on the nonce vowel categorizations.

Alternatively, negative effects of accent exposure may reflect system-wide recalibration, i.e., abstracting from experience to update categories shared across lexical items. We speculate that this type of abstraction can be modulated by phonetic properties of the accented vowel or lexical set in which the vowel occurs. Other factors may also condition the degree to which experienced variation generalizes to new situations. Episodic memory is crucial in the process: the relevant variation must be held in memory long enough to facilitate abstraction.

Several aspects of our findings are consistent with these speculations. Without Ncl exposure, Aus listeners did comparably poorly on Ncl TRAP, PALM and BATH vowels. After $\mathrm{Ncl}$ story exposure they adapted, but only to TRAP and PALM, not BATH, a restricted lexical set. Aus listeners are likely to know that BATH'S vowel is spoken like PALM by some speakers but TRAP by others (a variation heard in Australia), but listeners may be less likely to generalize such vowels to nonce words. Degree of phonetic variation within the target accent may also contribute to adaptation. Ncl CHOICE, FLEECE and PRICE vowels were all heard as Native-Like by Aus listeners. After $\mathrm{Ncl}$ exposure they showed improvement on CHOICE and FLEECE but not on PRICE, possibly due to the wider phonetic variation found in PRICE (see ellipses, Fig. 1).

\section{Conclusions}

We speculate that adaptation may initially destabilize the perceived vowel space for an unfamiliar accent as listeners seek to map the relations among other-accent vowels to the phonetic-phonological mappings of their own accent, thus optimizing access to their lexicon via words spoken in that accent.

\section{Acknowledgements}

This project was funded by ARC grant DP120104596. Many thanks to Meg Mundell, who created our Chicken Little story. 


\section{References}

[1] Cutler, A. 2010 Abstraction-based efficiency in the lexicon. Lab. Phon. 1, 301-318.

[2] Goldinger, S. 1998 Echoes of echoes? An episodic theory of lexical access. Psy. Rev. 105, 251-279.

[3] Goldinger, S. 2007 A complementary-systems approach to abstract and episodic speech perception. Proc $16^{\text {th }} I C P h S$ Saarbrucken 16, 49-54.

[4] Maye, J., Aslin, R. N., Tanenhaus, M. K. 2008 The weckud wetch of the wast: Lexical adaptation to a novel accent. $\mathrm{Cog} S c i$ 32, 543-562.

[5] Dahan, D., Drucker, S. J., Scarborough, R. A. 2008 Talker adaptation in speech perception: Adjusting the signal or the representations? Cognition 108, 710-718.

[6] Samuel, A.G., Kraljic, T. 2009 Perceptual learning for speech. Atten, Perc \& Psychophys 71, 1207-1218.

[7] Clopper, C. G., \& Bradlow, A. 2006 Effects of dialect variation on speech intelligibility in noise. J Acous Soc Am 119, 3424.

[8] Floccia, C., Goslin, J., Girard, F., Konopczynski, G. 2006 Does a regional accent perturb speech processing? J Exp Psy: Human Perc \& Perf 32, 1276-1293.

[9] Bradlow, A. R., Bent, T. 2008 Perceptual adaptation to nonnative speech. Cognition 106, 707-729.

[10] Clarke, C. M., Garrett, M. 2004 Rapid adaptation to foreign accented speech. J Acous Soc Am 116, 3647-3658.

[11] Evans, B. G., Iverson, P. 2004 Vowel normalization for accent: An investigation of best exemplar locations in northern and southern British English sentences. J Acous Soc Am 115, 352361.

[12] Cutler, A., Smits, R., Cooper, N. 2005 Vowel perception: Effects of non-native language vs. non-native dialect. Speech Commun $47,32-42$.

[13] Foulkes P. \& Docherty, G. (Eds) 1999 Urban Voices: Accent Studies in the British Isles London: Arnold.

[14] Wells, J. C. 1982 Accents of English, Vol. 3. Cambridge: Cambridge University Press.

[15] Cox, F. 2006 The acoustic characteristics of $/ \mathrm{hVd} /$ vowels in the speech of some Australian teenagers. Aus J Ling 26, 147-179.

[16] Cox, F., Palethorpe, S. 2007 Australian English. Int J Phon Assoc 37, 341-350.

[17] Best, C. T. 1995 A direct realist perspective on cross-language speech perception. In: W. Strange (ed), Speech Perception and Linguistic Experience. Timonium MD: York Press 167-200.

[18] Best, C. T., Tyler. M. D. 2007 Nonnative and second-language speech perception: Commonalities and complementarities. In: Munro, M., Bohn, O.-S. (eds) Second Language Speech Learning Amsterdam: Benjamins, 13-34.

[19] Lobanov, B. M. 1971 Classification of Russian vowels by different speakers. $J$ Acous Soc Am 49, 606-607.

[20] Fry, D.B., Abramson, A.S., Eimas, P.D. 1962 The identification and discrimination of synthetic vowels Lang Speech 5, 171-189.

[21] Bundgaard-Nielsen, R., Best, C. T., Kroos, C. H., Tyler, M. D. 2011 Vocabulary size matters: The assimilation of L2 Australian English vowels to L1 Japanese vowel categories. App Psycholing 32, 51-67.

[22] Johnson, K. 1997 Johnson, K. (1997) Speech perception without speaker normalization: An exemplar model. In K. Johnson \& J. Mullennix (eds) Talker Variability in Speech Processing. San Diego: Academic Press, 145-165. 